\title{
Kink propagation through disordered media
}

\author{
Sergey A. Gredeskul \\ Department of Physics, Ben Gurion University of Negev, P.O. Box 653, 84105 Beer Sheva, Israel
}

Yuri S. Kivshar*

Institute for Low Temperature Physics and Engineering, 47 Lenin Avenue, 310164 Kharkov, Ukraine and Departamento de Física Teórica I, Facultad de Ciencias Físicas, Universidad Complutense, E-28040 Madrid, Spain

Leonid K. Maslov

Institute for Low Temperature Physics and Engineering, 47 Lenin Avenue, 310164 Kharkov, Ukraine

Angel Sánchez

Departamento de Física Teórica I, Facultad de Ciencias Físicas, Universidad Complutense, E-28040 Madrid, Spain

Luis Vázquez

Departamento de Física Teórica I, Facultad de Ciencias Físicas, Universidad Complutense, E-28040 Madrid, Spain

and Instituto de Europa Oriental, Universidad Complutense, Somosaguas, E-28023 Madrid, Spain

(Received 7 January 1992)

\begin{abstract}
Using a collective-coordinate approach, we study kink propagation along nonlinear systems with local, randomly distributed inhomogeneities. We develop a general method to compute dynamical variable statistics which can be generalized to a number of soliton-bearing systems, and we apply it to the sine-Gordon equation as an example.
\end{abstract}

PACS number(s): 03.40.Kf, 42.25.Bs, 71.55.Jv

\section{INTRODUCTION}

Wave propagation in nonlinear disordered media has become an extensively studied subject in recent years [1]. In linear systems, disorder generally gives rise to Anderson localization, a phenomenon that is experienced also by a number of different waves, such as phonons, acoustic, and electromagnetic waves, etc. (see, e.g., Ref. [2]). Localization means that the transmission coefficient of a wave decays exponentially with the system length $L$, i.e., a positive number exists, the so-called localization length, $\lambda(k), k$ being the wave number, so that for $L \gg \lambda(k)$ very little transmission is allowed (see, e.g., [3]). Nonlinearity changes the features of wave scattering in disordered systems, bringing about qualitatively different effects. In stationary nonlinear problems, weak nonlinearity acting against disorder induces variations of the length dependence of the transmission coefficient: it still goes to zero as the system size increases, but according to a power law $[4,5]$ instead of the exponential one proper to the linear case. Nonlinearity may also produce multistability in the wave transmission through a disordered slab [4-6]. In nonstationary problems, nonlinearity may lead to modulational instability, which is enhanced by disorder $[7,8]$. The modulational instability is important because it is the factor that causes the formation of envelope solitons instead of linear waves.
The most remarkable manifestation of the influence of nonlinearity on disordered systems is related to the fact that many nonlinear models are able to support undistorted propagation of localized waves, i.e., solitons. Nonlinearity yields a sufficient improvement of the transmission through the disordered medium only when it contributes to create soliton pulses [9-11]. Solitons are far more robust than linear waves, and that is the reason why their structure acts against localization. Therefore, solitons have to give a nonzero contribution to the total transmission coefficient. As is well known, there are solitons of three general types: dynamical solitons, envelope solitons, and (topological) kinks. The amplitude of a dynamical soliton is proportional to its velocity, and, when propagating along a disordered system, their scattering is similar to that of a linear wave packet [9]. Envelope solitons are two-parametrical ones, and they exhibit a quite more complicated behavior. As was shown by the authors [11] for envelope solitons, localization effects vanish drastically above a certain threshold in the strength of the nonlinearity. As we stated above, this property means that such solitons will provide a nonzero wave transmission coefficient, even for distances much longer than the localization length. The last type of solitary waves is that of topological kinks, which exist only in nonlinear systems with two or more equivalent ground states, i.e., when nonlinearity is not small. In many 
cases, kink dynamics can be described in the framework of a collective-coordinate approach, when the evolution of the kink parameters is similar to the evolution of an effective particle (see [12-16] and references therein), the particle coordinate being the kink center position. Such a simplification holds because of an exponentially small radiative loss of the kink in the spatially inhomogeneous medium (see, e.g., [14]); under this condition, one can use the collective-coordinate formalism with high accuracy (see [17] for an example). However, this technique is no longer reliable if the medium parameters are nonstationary: then, perturbation-induced emission is not at all small in this case.

The present paper is aimed to study kink propagation in a disordered medium using the sine-Gordon (SG) model as an example. Starting from the standard collective-coordinate treatment, we reduce the model to a simple equation for the kink coordinate that includes an effective random potential. To deal with the soobtained equation, we elaborate a statistical method to compute directly the mean values of the kink parameters. This procedure is rather general, and it may be useful in other similar problems, such as, e.g., propagation of kinks along spatially disordered $\phi^{4}$ [18], double-SG [19], or generalized-SG [20] models, as well as for other nonlinear systems supporting topological kinks. Thus, the paper is organized as follows: in Sec. II we present the model and derive the equation for the kink collective coordinate, with an effective random potential accounting for the effects of disorder. Section III is devoted to the statistical procedure, developed to describe the kink propagation in the system by obtaining exact formulas for the mean kink parameters. In Sec. IV we numericallly evaluate these formulas for some cases and discuss the validity and interest of our results in view of the outcome. Finally, Sec. V collects our results and conclusions.

\section{MODEL: EFFECTIVE EQUATION FOR THE KINK COORDINATE}

Let us consider a kink-bearing system in the presence of a disordered lattice of impurities. To be specific, and to make calculations simpler, let us choose the $S G$ model as a particular realization of this problem, described by the following equation for the wave field $\phi(x, t)$ :

$$
\phi_{t t}-\phi_{x x}+\sin \phi=-f(x) \sin \phi,
$$

where the subscripts $t$ and $x$ stand for partial derivatives in time and spatial coordinates, respectively, and units are such that the equation is already in dimensionless form. In the simplest model of a disordered system, the function $f(x)$ may be taken to be a sum of $\delta$ functions,

$$
f(x)=\sum_{n} \epsilon_{n} \delta\left(x-a_{n}\right),
$$

where the numbers $\epsilon_{\mathfrak{n}}$ and $a_{n}$ are randomly chosen. We shall assume further that the distances $b_{n}=a_{n+1}-a_{n}$ are identically distributed random variables with probability density

$$
p(b)=b_{0}^{-1} \exp \left(-b / b_{0}\right)
$$

and, for simplicity, we shall consider only the case when the strength of all the impurities is the same, although the method can be straightforwardly generalized to treat more general perturbations.

In the absence of any inhomogeneity, Eq. (2.1) has a topologically stable solution in the form of a kink given by

$$
\phi_{k}(x, t)=4 \tan ^{-1} \exp \left[\sigma \frac{x-X}{\sqrt{1-V^{2}}}\right],
$$

where $X=V t-X_{0}$ is the kink coordinate, $V$ is its velacity, and $\sigma$ is the kink polarity, which can take one of the two values \pm 1 corresponding, respectively, to a kink or an antikink. Furthermore, the SG equation then has a conserved momentum,

$$
P \equiv-\int_{-\infty}^{\infty} d x \phi_{t} \phi_{x} .
$$

For the particular solution (2.4), Eq. (2.5) is cast into the well-known relativistic expression $P=8 \mathrm{~V} / \sqrt{1-V^{2}}$.

Once we have mentioned the features of the unperturbed model which will be used later, we shall move to the disordered case. We shall study it by means of the collective-coordinate approach for the kink. This means that our main assumption is to suppose that the kink parameters are slowly varying functions of time, so that the essential properties of the kink dynamics can be analyzed in the framework of a set of ordinary differential equations for these parameters. Other degrees of freedom of the SG system, such as those related to the emission of linear waves generated by the perturbation, are assumed to have negligible variations with respect to that of the kink collective coordinate.

The easiest way to derive the equations for the kink collective coordinate is to use conservation laws. Having in mind the definition (2.5) and the boundary conditions $\phi \rightarrow 0(\bmod 2 \pi), \phi_{x} \rightarrow 0$ when $|x| \rightarrow \infty$, it is straightforward to show that in the presence of impurities the momentum $P$ changes according to

$$
\frac{d P}{d t}=-\epsilon \sum_{n} \cdot \int_{-\infty}^{\infty} d x \delta\left(x-a_{n}\right)(\cos \phi)_{x} .
$$

If the perturbation strength $\epsilon$ is small, only kinks with momentum $P \sim \epsilon$ will be affected by the impurity lattice, and hence we can restrict ourselves to the so-called nonrelativistic limit, $V^{2} \ll 1$. In this limit, using Eq. (2.4) it is not difficult to obtain from Eq. (2.6) the following expression for the kink coordinate $X$ :

$$
\frac{d^{2} X}{d t^{2}}=-\frac{d}{d X} U(X)
$$

where

$$
\begin{aligned}
& U(X)=\sum_{n} u_{n}(X) ; u_{n}(X) \equiv u\left(X-a_{n}\right) \\
& u(X) \equiv \frac{\epsilon}{4 \cosh ^{2} X} .
\end{aligned}
$$


Here we have assumed $P \simeq 8 V \simeq 8 d X / d t$, which is valid for $V^{2} \ll 1$. Thus, in the collective-coordinate framework, the motion of the SG kink can be interpreted as the motion of a nonrelativistic particle with unit mass in an effective, random potential defined by Eq. (2.8). Of course, this result is similar to others coming out from the study of regular perturbations $[12,14,21]$.

\section{STATISTICAR ANALYSIS OF THE KINK PROPAGATION}

To simplify the computation of the kink statistics, let us consider the potential $U(X)$ as a finite one, of width $\Delta$, i.e., we introduce the cutoff

$$
U(X)=0 \text { if }|x|>\frac{\Delta}{2} .
$$

Now, we assume that the distances [see Fig. 1(a)]

$$
y_{n} \equiv b_{n}-\Delta=a_{n+1}-a_{n}-\Delta>0
$$

are identically distributed random values with probability density [cf. Eq. (2.3)]

$$
p(y)=y_{0}^{-1} \exp \left(-y / y_{0}\right) \text {. }
$$

Here $y_{0}$ represents the mean length of empty intervals between effective scatterer potentials (3.1). From the rigorous point of view, this probability distribution is not the exact one which follows from Eq. (2.3). But in the dilute-lattice limit, $y_{0} \gg \Delta$, this difference is negligible if one is not interested in the effects induced by overlapping of the potentials $U(X)$. Notice also that in this limit the relation $y_{0} \simeq b_{0}$ is valid too.

Our goal will be the calculation of the mean values

(a)

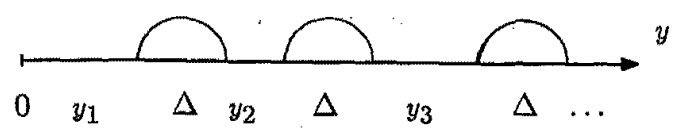

Spatial picture

(b)

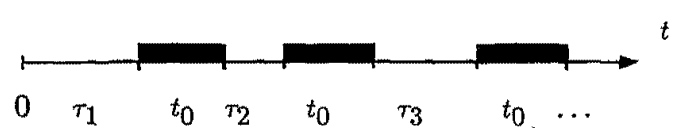

Time picture

FIG. 1. The effective potential $U(x)$ corresponding to a disordered lattice of point impurities (a), and its representation in the time scale (b). In (a), scatterers are represented by the curves above the line; in (b), scatterers are shown as black intervals. (and their dispersions) of the kink parameters, namely the mean coordinate and velocity of the kink at each time instant $t$. To this end, we found it much more convenient to describe the process in terms of the time distribution of the scatterers instead of their spatial one; i.e., more strictly speaking, we study the problem by means of the time intervals $\tau_{n}$ elapsed when the kink is between the $n$th and $(n+1)$ th scatterers $(n=1,2, \ldots)$. Each one of these intervals will be separated by equal times in which the kink travels through an impurity with variable velocity, whose value is

$$
t_{0}=\int_{-\Delta / 2}^{\Delta / 2} \frac{d X}{\dot{X}(X)}=\int_{-\Delta / 2}^{\Delta / 2} \frac{d X}{\sqrt{V_{0}^{2}-2 U(X)}}
$$

$\dot{X}(X) \equiv d X / d t$ being the local kink velocity, and $V_{0}$ being the velocity of the kink in the scatterer-free regions. With respect to the time intervals $\tau_{n}$, they are equal to $y_{n} / V_{0}$, and subsequently they are governed by the probability density

$$
p(\tau)=\tau_{0}^{-1} \exp \left(-\tau / \tau_{0}\right)
$$

where $\tau_{0} \equiv y_{0} / V_{0}$, and $\tau \in[0, \infty]$. Therefore, instead of the succession of the random spatial intervals of the form $y_{1}, \Delta, y_{2}, \Delta, y_{3}, \ldots, \Delta, y_{n}, \Delta, y_{n+1}, \ldots$, we shall consider the corresponding succession $\tau_{1}, t_{0}, \tau_{2}, t_{0}$, $\tau_{3}, \ldots, t_{0}, \tau_{n}, t_{0}, \tau_{n+1}, \ldots$ This is graphically depicted in Figs. 1(a) and 1(b).

The next point to take into account is that there are configurations of scatterers of two different types. Configurations of the first kind (referred to as configurations I) are defined as those in which the interval $[0, t]$ includes $n$ empty intervals $\tau_{1}, \ldots, \tau_{n}$, so that at the moment $t$ the kink moves in the region where the potential is zero, i.e., freely [see Fig. 2(a)]. The probability $P_{n}(t)$ of finding

(a)

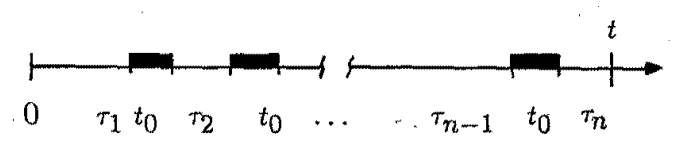

Configuration I

(b)

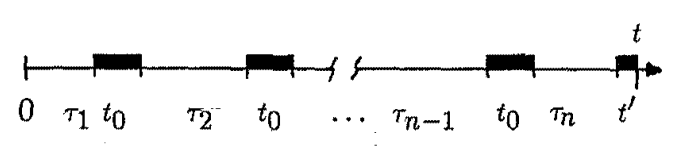

\section{Configuration II}

FIG. 2. Different configurations of scatterers: configuration I (a) and configuration II (b) (see details in the text). The instant $t$ is the current time. Scatterers are shown in black as in Fig. 1. Notice that in (b), the last scatterer is not completely contained in the interval. The kink travels through it during a time $t^{\prime}$. 
lattice configurations I can be calculated as

$$
P_{n}(t)=\left\langle\Theta\left(\tau^{\prime}\right) \Theta\left(\tau_{n+1}-\tau^{\prime}\right)\right\rangle
$$

with

$$
\tau^{\prime} \equiv t-n t_{0}-\sum_{j=1}^{n} \tau_{j}
$$

$\theta(\tau)$ being the Heaviside step function, and the angular brackets in Eq. (3.6) stand for the average over the $n+1$ random variables $\tau_{1}, \ldots, \tau_{n+1}$, all of them having probability density (3.5). Equation (3.6) has a simple physical interpretation. The first $\theta$ function means that $n$ scatterers are contained in the interval $[0, X]$, where $X=X(t)$, and the second one accounts for the fact that there are no other scatterers in the interval. After some straightforward calculations using the probability density (3.5), we obtain for $P_{n}(t)$ the exact result

$$
\begin{array}{r}
P_{n}(t)=\frac{1}{n !}\left(\frac{t-n t_{0}}{\tau_{0}}\right)^{n} \exp \left(-\frac{t-n t_{0}}{\tau_{0}}\right), \\
n=0,1, \ldots, N,
\end{array}
$$

where $N \equiv\left[t / t_{0}\right]$ and $[z]$ stands for the operation of truncating $z$ to its integer part.

Configurations of the second type (hereafter referred to as configuration II) consist of $n$ empty intervals and their corresponding impurities as in configuration I, plus one extra scatterer along which the kink travels during the interval $t^{\prime}<t_{0}$, i.e., a time less than that neeeded for the kink to go through one scatterer [see Fig. 2(b)]. The probability density $p_{n}\left(t \mid t^{\prime}\right)$ for such configurations, with fixed $n$ and $t^{\prime}$, is given by

$p_{n}\left(t \mid t^{\prime}\right)=\left\langle\delta\left(\tau^{\prime}-t^{\prime}-\tau_{n+1}\right) \theta\left(t_{0}-t^{\prime}\right) \theta\left(t^{\prime}\right)\right\rangle$,

where $\tau^{\prime}$ is the same as in Eq. (3.7). The appearance of the $\delta$ function in this expression is connected with the fact that, at the moment $t$, the kink has moved during a time $t^{\prime}$ [it is important to realize that $p_{n}\left(t \mid t^{\prime}\right)$ is a function of $t^{\prime}$ and $t$ is a fixed parameter] under the influence of the last [the $(n+1) t h]$ scatterer, whereas the Heaviside functions ensure that this time interval $t^{\prime}$ is positive and less than $t_{0}$. As above, after computing the corresponding integral, we obtain the result

$$
\begin{aligned}
p_{n}\left(t \mid t^{\prime}\right)= & \frac{1}{\tau_{0} n !}\left(\frac{t-t^{\prime}-n t_{0}}{\tau_{0}}\right)^{n} \\
& \times \exp \left(-\frac{t-t^{\prime}-n t_{0}}{\tau_{0}}\right) \Theta\left(t_{0}-t^{\prime}\right) \Theta\left(t^{\prime}\right) .
\end{aligned}
$$

Finally, concerning the normalization of the probability (3.8) and the probability density (3.10), they have to verify the relationship

$$
\begin{aligned}
& \sum_{n=0}^{N} P_{n}(t)+\sum_{n=0}^{N \cdots 1} \int_{0}^{t_{0}} d t^{\prime} p_{n}\left(t \mid t^{\prime}\right) \\
& +\int_{0}^{t_{0}\left\{t / t_{0}\right\}} d t^{\prime} p_{N}\left(t \mid t^{\prime}\right)=1
\end{aligned}
$$

where $\{z\} \equiv z-[z]$ stands for the fractional part of $x$. It is easy to check, taking into account Eqs. (3.6), (3.8), (3.9), and (3.10), that condition (3.11) is fulfilled.

Now, we address the next step. It is clear that, following the same line of reasoning, any dynamical variable $F(t)$ can be considered either as a function of $n$, i.e., $F_{n}(t)$, for configuration $\mathrm{I}$, or as a function of $n$ and $t^{\prime}$, $F_{n}\left(t \mid t^{\prime}\right)$, for configuration II. For instance, the kink coordinate $X(t)$ can be expressed as

$$
\begin{aligned}
& X_{n}(t)=V_{0} t-n \xi_{0} \text { for configuration } \mathrm{I}, \\
& X_{n}\left(t \mid t^{\prime}\right)=V_{0} t-n \xi_{0}-\xi^{\prime} \text { for configuration II, }
\end{aligned}
$$

where we have introduced the notations

$$
\xi^{\prime} \equiv V_{0} t^{\prime}-x^{\prime}, \quad \xi_{0} \equiv V_{0} t_{0}-\Delta,
$$

and $t^{\prime}$ is connected to $x^{\prime}$ by

$$
t^{\prime}=\int_{-\Delta / 2}^{-\Delta / 2+x^{\prime}} \frac{d X}{X(X)}
$$

so that $t^{\prime}\left(x^{\prime}\right)$ is the part of the last scatterer (in the terporal scale) that is contained in the whole motion interval $[0, t]$, or equivalently $[0, x(t)]$. Analogously, the kink velocity at the time instant $t$ may be written in the form

$$
\begin{aligned}
& V_{n}(t)=V_{0} \text { for configuration I, } \\
& V_{n}\left(t \mid t^{\prime}\right)=v\left(t^{\prime}\right) \equiv v\left[x^{\prime}\left(t^{\prime}\right)\right] \text { for configuration II. }
\end{aligned}
$$

We can now write the main result of this section. Recalling the probability (3.8) and the probability density (3.10) and writing time-dependent functions $F(t)$ as explained above, we can compute their mean value according to

$$
\begin{aligned}
<F(t)>= & \sum_{n=0}^{N} F_{n}(t) P_{n}(t) \\
& +\sum_{n=0}^{N-1} \int_{0}^{t_{0}} d t^{\prime} F_{n}\left(t \mid t^{\prime}\right) p_{n}\left(t \mid t^{\prime}\right) \\
& +\int_{0}^{t_{0}\left\{t / t_{0}\right\}} d t^{\prime} F_{N}\left(t \mid t^{\prime}\right) p_{N}\left(t \mid t^{\prime}\right)
\end{aligned}
$$

where $N \equiv N(t)=\left[t / t_{0}\right]$. We shall subsequently apply this equation to the problem we posed in Sec. II.

\section{RESULTS AND DISCUSSION}

In this section, we shall compute the mean position of a SG kink propagating along the inhomogeneous model we described above. To this end, and in accordance with the hypothesis (3.1) we have done at the beginning of Sec. III, we shall slightly modify the potential (2.8), truncating it to

$$
U(X)= \begin{cases}(\epsilon / 4) \operatorname{sech}^{2} X & \text { if }|X|<\Delta / 2 \\ 0 & \text { if }|X|>\Delta / 2\end{cases}
$$

Notice that this truncation is actually not very relevant, because the effective potential is an exponentially decaying one. We are now ready to carry out the procedure we 
developed in the preceding section in an explicit fashion; thus, we have

$$
\begin{aligned}
& t_{0}=\frac{2}{V_{0}} \sinh ^{-1}\left(\frac{2 V_{0}}{\sqrt{4 V_{0}^{2}-\epsilon}} \sinh \frac{\Delta}{2}\right) \\
& t^{\prime}\left(x^{\prime}\right)=\frac{1}{2} t_{0}-\frac{1}{V_{0}} \sinh ^{-1}\left[\frac{2 V_{0}}{\sqrt{4 V_{0}^{2}-\epsilon}} \sinh \left(\frac{\Delta}{2}-x^{\prime}\right)\right] \\
& x^{\prime}\left(t^{\prime}\right)=\frac{1}{2} \Delta-\sinh ^{-1}\left(\frac{\sqrt{4 V_{0}^{2}-\epsilon}}{2 V_{0}} \sinh \left[V_{0}\left(\frac{1}{2} t_{0}-t^{\prime}\right)\right]\right)
\end{aligned}
$$

It is now straightforward to use Eqs. (4.1) to (4.4) to compute the mean parameters of the kink moving in the disordered potential, substituting them in the main formulas (3.8), (3.10), and (3.18). The so-derived expressions are actually very cumbersome and we shall not write them down here. Instead, what we have done is to compute their values numerically for some parameter $\left(\epsilon, V_{0}\right)$ values, in order to understand their physical meaning. In this numerical calculation, we took the parameter $\Delta$ to be such that $(\epsilon / 4) \operatorname{sech}^{2}(\Delta / 2)<10^{-4}$, so that the finite length of the effective potential is a very good approximation of the real problem. All the integrals were computed up to the same precision using efficient standard routines [22].

Let us begin with the discussion of the numerical results by defining the magnitude $L \equiv\langle X\rangle-V_{\text {as }} t$, which we shall call the asymptotic difference and where $V_{\text {as }}$ is the asymptotic velocity of the kink, given by

$$
\begin{aligned}
V_{\mathrm{as}} & \equiv \lim _{t \rightarrow \infty} \frac{V_{0} t-n \xi_{0}}{t} \\
& =V_{0}-\left\langle\frac{n}{t}\right\rangle \xi_{0}=V_{0}-\frac{\xi_{0}}{\tau_{0}+t_{0}}=\frac{V_{0} \tau_{0}+\Delta}{\tau_{0}+t_{0}}
\end{aligned}
$$

where the limit has to be taken having in mind the condition $n / t \rightarrow\langle n / t\rangle=\left(\tau_{0}+t_{0}\right)^{-1}$ when $t$ (and of course $n)$ go to infinity. The quantity $L$ then measures how far the kink behavior is from the asymptotic one, and is thus a good magnitude to characterize the calculations we describe below.

Our numerical results are summarized in Figs. 3-6. All of them show the asymptotic difference relative to the traveled distance $X(t)$, in order to decide whether we have reached the limit value of $V_{a s}$ or not. The impurity strength is always taken to be $\epsilon=0.1$. In Fig. 3 , we show the early stages of a typical kink trajectory through the disordered medium. We can see the very large initial deviation from the asymptotic behavior as predicted by Eq. (4.5), which happens for times less than that needed to cross one scatterer, $t_{0}$ (with the parameters chosen for Fig. $3, t_{0}=24.1$ ). After that, the evolution of $L$ becomes monotonic, steadily decreasing, at a scale which is not visible in the scale of Fig. 3. This asymptotic behavior is shown in Fig. 4, in which we plot the evolution for a case with the same parameters of Fig. 3 but for much longer times. It is seen that at times around those needed to cross two impurities, $L$ reaches very small [again, as

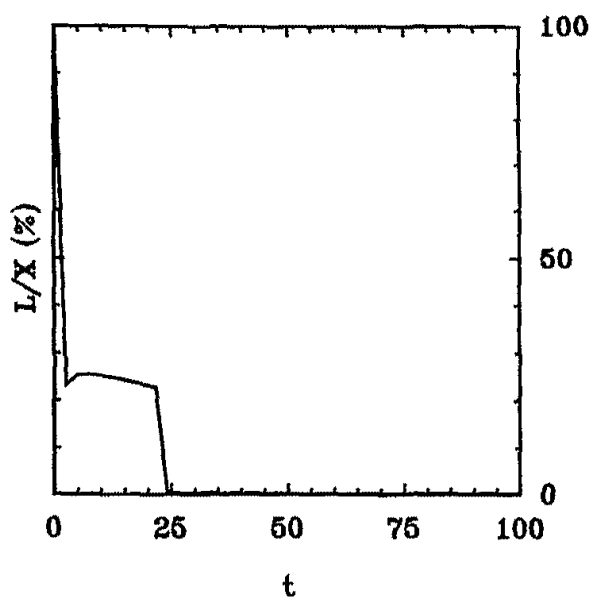

FIG. 3. Asymptotic difference $L$ as a percentage of the position $X(t)$ vs time [i.e., $100 \times L(t) / X(t)$ ], when $\epsilon=0.1$, $V_{0}=0.3$ (which implies $t_{0}=24.1$ ), and $\tau_{0}=3 t_{0}$.

compared to the amount of space already traveled, $X(t)]$ values and continues decreasing towards zero in a monotonic fashion. As to the peaks and discontinuities appearing in Fig. 3 and also in subsequent ones, we believe that they come from the transient behavior of $L$ before reaching the asymptotic regime, as well as from the resolution (usually around 100 points) of our calculation. However, they are not very relevant, because they arise when the kink has traveled over at most two scatterers, and we obviously do not expect our statistics to be useful at that short time (and short number of scatterers crossed).

From our numerical computation of $\langle X(t)\rangle$ and $L$ using Eqs. (3.12), (3.13), and (3.18), we can conclude that our statistical procedure is very useful after the kink has crossed the first impurities, because at that time we are already in the asymptotic regime and the simple estima-

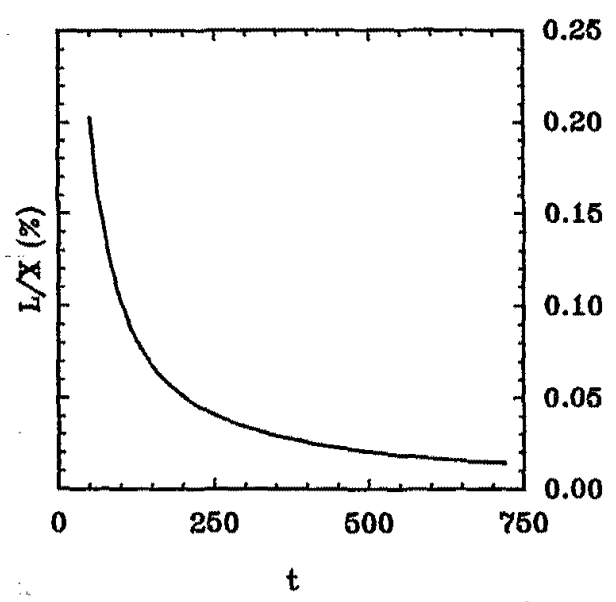

FIG. 4. Same as in Fig. 3. In this plot we show an enlargement of the asymptotic part up to very long times. The value of $L$ is seen to steadily decrease. 


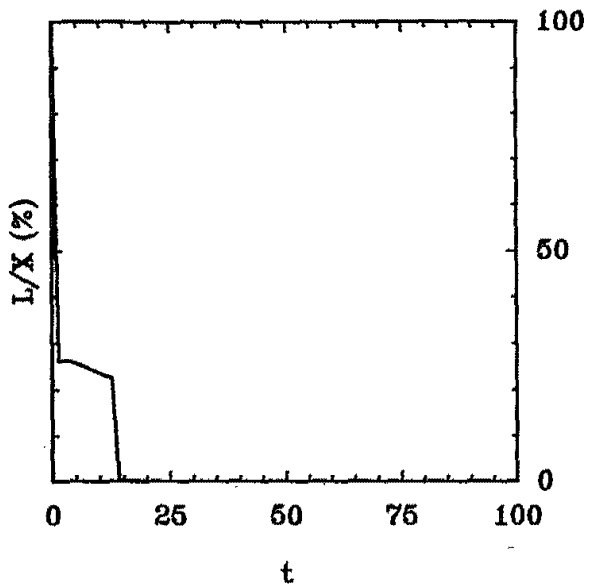

FIG. 5. As in Fig. 3 , but in this plot $V_{0}=0.5$ (and hence $t_{0}=14.02$ ). We see that the asymptotic behavior starts always at a point around $t_{0}$.

tion of its position through the expression $X(t) \simeq V_{\text {as }} t$ gives fairly good results. The validity of the asymptotic formula depends on the time $t_{0}$ to cross one impurity, as can be seen from Fig. 5, where $V_{0}=0.5$ and then $t_{0}=14.02$; in this plot it is again observed how large deviations happen only before $t_{0}$. On the other hand, the time for the evolution of $L$ does not exhibit any influence of the mean empty interval, $\tau_{0}$, as is appreciated from Fig. 6, which should be compared to Fig. 3, whose parameters are the same except for $\tau_{0}$. Thus, we are led to conclude that the important parameter to decide whether we have reached the asymptotic regime or not is the impurity potential length, while the influence of the other ones is not very noticeable.

\section{CONCLUSIONS}

We have studied analytically the problem of kink propagation through a disordered medium, where disorder was modeled by a lattice of $\delta$-like impurities with equal intensities but random positions. In the framework of the collective-variable approach, the equation for the kink coordinate turns out to be that of an effective particle in an effective disordered potential. Because of this, the Fokker-Planck-equation methods cannot be straightforwardly applied to our problem (notice that the potential is not a Markov one) and we have had to develop a more sophisticated statistical procedure to compute the mean features of kink propagation. This procedure has allowed us to obtain exact expressions for the SG model, although, due to their cumbersome form, we have investigated them numerically. By this means, we have checked how the mean kink position evolves with time, and we have concluded that after a time of the order of the one needed to cross about two impurities, the asymptotic estimations for the kink velocity work very well, whatever the mean times between impurities corresponding to the initial velocity are. This is very important in order to

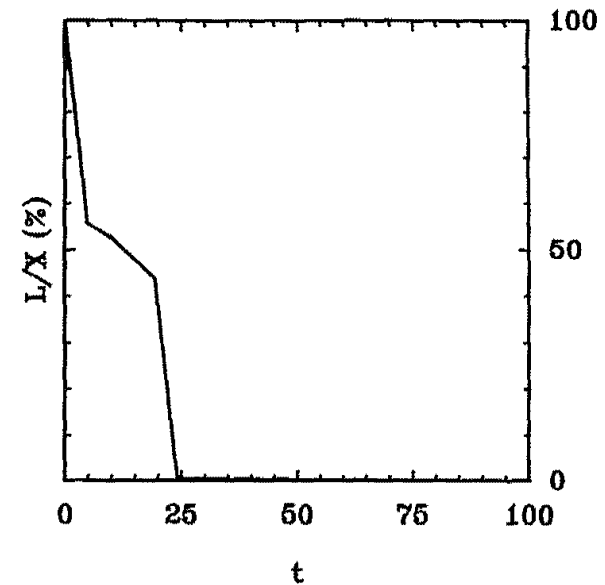

FIG. 6. As in Fig. 3, but now the mean time interval between impurities has been changed to be $\tau_{0}=t_{0}$. Notice that the asymptotic behavior starts at the same point as in Fig. 3.

give practical usefulness to our results.

It is clear that the procedure we have worked out to obtain the mean kink parameters is quite general, and likely to apply to a number of different soliton-bearing systems, such as those we mentioned in the Introduction ( $\phi^{4}$ [18], double SG [19], generalized SG [20]). The reason is that they also allow for kink propagation and the collective-coordinate approximation is as good as in the SG equation; then, the single-scatterer effective potential can be computed and the formalism we present here applies inmediately. We would like to point out also that to present a global picture of kink propagation in disordered media, it is necessary to compare the results we have obtained to numerical simulations of the ordinary differential equations for the kink collective coordinate (2.7) and (2.8), as well as to direct numerical simulations of the model as described by the corresponding partial differential equation (2.1) with the perturbation (2.2). This numerical work is currently in progress, and preliminary results for the collective coordinate equations show good agreement with the predictions of this work. A detailed comparison of the present results to simulations will be published elsewhere.

\section{ACKNOWLEDGMENTS}

S.A.G. and Y.S.K. want to thank Luis Vázquez and the Departamento de Física Teórica I of the Universidad Complutense for hospitality during their stay in Madrid, whereas A.S. thanks Alan Bishop and also the Los Alamos National Laboratory for hospitality during some stages of this work. We are grateful to J. M. Márquez for the use of the VAX 9000 computer of the Universidad Complutense. A.S. and L.V. are partially supported by the Comisión Interministerial de Ciencia y Tecnologia (CICyT) of Spain through project MAT900544, and S.A.G. is partially supported by a grant from the Israeli Ministry for Science and Development. 
* Present address: Institut für Theoretische Physik I, Heinrich-Heine-Universität Düsseldorf, Universitätstrasse 1, D-4000 Düsseldorf, Germany.

[1] For reviews, see A. Sánchez and L. Vázquez, Int. J. Mod. Phys. B 5, 2825 (1991); S. A. Gredeskul and Yu. S. Kivshar, Phys. Rep. (to be published).

[2] Scattering and Localization of Classical Waves, edited by Ping Sheng (World Scientific, Singapore, 1990).

[3] I. M. Lifshitz, S. A. Gredeskul, and L. A. Pastur, Introduction to the Theory of Disordered Systems (Wiley, New York, 1988).

[4] P. Devillard and B. Souillard, J. Stat. Phys. 43,423 (1986).

[5] B. Douçot and R. Rammal, Europhys. Lett. 3, 969 (1987); J. Phys. (París) 48, 509 (1987).

[6] R. Knapp, 'G. Papanicolau, and B. White, in Disorder and Nonlinearity, edited by A. R. Bishop, D. K. Campbell, and St. Pnevmatikos, Springer Proceedings in Physics Vol. 39 (Springer, Berlin, 1989); J. Stat. Phys. 63, 567 (1991).

[7] M. Peyrard and A. R. Bishop, in Nonlinear Coherent Structures, edited by M. Barthes and J. Léon, Lecture Notes in Physics Vol. 353 (Springer, Berlin, 1990).

[8] Yu. S. Kivshar, in Nonlinearity with Disorder, edited by F. Kh. Abdullaev, A. R. Bishop, and St. Pnevmatikos, Springer Proceedings in Physics (Springer, Berlin, 1992).

[9] Q. Li, C. M. Soukoulis, St. Pnevmatikos, and E. N. Economou, Phys. Rev. B 37, 3534 (1988).

[10] J. G. Caputo, A. C. Newell, and M. Shelley, in Proceedings of "Workshop on Stimulated Nonlinear Effects in
Josephson Devices," edited by M. Russo and G. Costabile (World Scientific, Singapore, 1989).

[11] Yu. S. Kivshar, S. A. Gredeskul, A. Sánchez, and L. Vázquez, Phys. Rev. Lett. 64, 1693 (1990); Waves Rand. Media (to be published).

[12] D. W. McLaughlin and A. C. Scott, Phys. Rev. A 18, 1652 (1978).

[13] P. J. Pascual, L. Vázquez, A: R. Bishop, and St. Pnevmatikos, in Disorder and Nonlinearity (Ref. [6]).

[14] Yu. S. Kivshar and B. A. Malomed, Rev. Mod. Phys. 61, 763 (1989).

[15] A. Sánchez, R. Scharf, A. R. Bishop, and L. Vázquez, Phys. Rev. A 45, 6031 (1992); R. Scharf, Yu. S. Kivshar, A. Sánchez and A. R. Bishop, ibid, 45, R5369 (1992).

[16] R. Boesch and C. Willis, Phys. Rep. (to be published).

[17] Yu. S. Kivshar, Z. Fei, and L. Vázquez, Phys. Rev. Lett. 67, 1177 (1991); Z. Fei, Yu. S. Kivshar, and L. Vázquez (unpublished).

[18] J. A. Krumhansl and J. R. Schrieffer, Phys. Rev. B 11, 3535 (1975); S. Aubry, J. Chem. Phys. 62, 3217 (1975); 64, 3392 (1976).

[19] D. K. Campbell, M. Peyrard, and P. Sodano, Physica. 19D, 165 (1986).

[20] M. Peyrard and D. K. Campbell, Physica 9D, 33 (1983).

[21] M. B. Fogel, S. E. Trullinger, A. R. Bishop, and J. A. Krumhansl, Phys. Rev. Lett. 36, 1411 (1976); Phys. Rev. B 15, 1578 (1977).

[22] W. H. Press, B. P. Flannery, S. A. Teukolsky, and W. T. Vetterling, Numerical Recipes in Fortran, 2nd ed. (Cam-

... bridge University Press, New York, 1989), pp. 110-114. 